\title{
Neural Stem Cell Detection, Characterization, and Age- Related Changes in the Subventricular Zone of Mice
}

\author{
Alexander Y. Maslov, ${ }^{1}$ Tara A. Barone, ${ }^{2}$ Robert J. Plunkett, ${ }^{2}$ and Steven C. Pruitt ${ }^{1}$ \\ Departments of ${ }^{1}$ Molecular and Cellular Biology and ${ }^{2}$ Surgical Oncology, Roswell Park Cancer Institute, Buffalo, New York 14263
}

\begin{abstract}
The mammalian brain contains neural stem cells (NSCs) that allow continued neurogenesis throughout the life of the animal. However, neurogenesis is known to decline during aging and, to the extent that neurogenesis is required for normal CNS function, this may contribute to neurodegenerative disease. Decreased neurogenesis could result from loss of NSCs or dysfunction at some later step, and distinguishing these possibilities is important for understanding the cause of the decline. However, because of the inability to distinguish NSCs from their rapidly dividing progeny in situ, it has not been possible to quantitatively assess the NSC populations in young and old animals. In this report we show that the G1 phase-specific expression of the replication factor $\mathrm{Mcm} 2$ is a useful marker for detecting slowly cycling putative NSCs in situ and confirm the identity of these cells using both cytosine $\beta$-D-arabinofuranoside (Ara-C) treatment and a double nucleoside analog-labeling technique. The ability to distinguish NSCs from proliferative progenitors has allowed characterization of the expression of several markers including Nestin, Musashi, and GFAP in these different cell types. Furthermore, comparison of the NSC populations in the subventricular zones of young (2-4 months) and old (24-26 months) mice demonstrates an approximately twofold reduction in the older mice. A similar twofold reduction is also observed in the number of neurospheres recovered in culture from old relative to young animals. The reduction in the neural stem cell population documented here is sufficient to account for the reduced level of neurogenesis in old animals.
\end{abstract}

Key words: neural stem cell; neurogenesis; aging; Mcm2 replication factor; subventricular zone; neurosphere

\section{Introduction}

Dividing cells persist within the brains of adult mammals, including rodents (Altman and Das, 1965), primates (Pencea et al., 2001), and humans (Eriksson et al., 1998; Kukekov et al., 1999), in both the subventricular zone (SVZ) of the lateral ventricles and the dentate gyrus of the hippocampus. Within the SVZ, three lines of evidence suggest that proliferation is maintained through the presence of neural stem cells (NSCs). First, cells that retain the ability to proliferate for long periods, but differentiate toward neuronal lineages on growth factor withdrawal, can be cultured from adult mammalian brains, including mice (Reynolds and Weiss, 1992) and humans (Kukekov et al., 1999). Second, retroviral tracing studies demonstrate that a subpopulation of marked cells remain within the proliferative zones and continue to divide (Morshead et al., 1998). Both retroviral tracing and nucleoside labeling studies show that dividing cells within the SVZ can be separated into two populations based on their cycles: a rapidly dividing population $(12 \mathrm{hr})$ and a slowly dividing population (15 d; Morshead et al., 1994, 1998; Tropepe et al., 1997; Doetsch et al., 1999a). Finally, after elimination of the rapidly dividing cells by

Received Aug. 1, 2003; revised Dec. 6, 2003; accepted Dec. 8, 2003.

This work was supported by National Institutes of Health Grants AG020946 and AG019863 to SCP. We thank Joel Huberman and Ivan Todorov for providing anti-Mcm2 antibody used in pilot studies leading to the present work and William Burhans for helpful discussions.

Correspondence should be addressed to Steven C. Pruitt, Department of Molecular and Cellular Biology, Roswell Park Cancer Institute, Buffalo, NY 14263. E-mail: steven.pruitt@roswellpark.org.

DOI:10.1523/JNEUROSCI.4608-03.2004

Copyright $\odot 2004$ Society for Neuroscience $\quad$ 0270-6474/04/241726-08\$15.00/0 local application of Ara-C, animals reconstitute these cells on its withdrawal (Doetsch et al., 1999b). This observation is consistent with their derivation from the slowly cycling population of cells. These studies demonstrate that neurogenesis within the SVZ is maintained through the asymmetric division of slowly cycling NSCs which, in addition to maintaining themselves, results in proliferative progenitors. The proliferative progenitors then divide rapidly but for a limited number of cycles. By this mechanism, the single cell type that retains the potential to proliferate in the adult brain over long periods is the NSC.

The localization of expression of several proteins to the region of the SVZ has been demonstrated previously. These include Nestin, Musashi, and GFAP, and each has been proposed to allow identification of NSCs and/or proliferative progenitors in different studies (Doetsch et al., 1999a; Kaneko et al., 2000; Rietze et al., 2001). However, these markers do not colocalize, and none of them is known to be expressed only within the NSC compartment.

Aging results in a factor of two decline in neurogenesis, as measured by 5-bromo-2'-deoxyuridine (BrdU) incorporation in the SVZ (Tropepe et al., 1997). This decline could result from changes in cell proliferation or survival in either the NSC or proliferative progenitor compartments. However, comparison of the numbers of NSCs and proliferative progenitors in young and old animals directly in situ has not been possible because of a lack of markers with which to distinguish these populations.

In the present study two alternative methods for identifying NSCs based on their prolonged cycling time are developed. First, 
the expression of the G1-phase cell cycle marker Mcm2 (Stoeber et al., 2001) is used. $\mathrm{Mcm} 2$ is part of a prereplicative complex that forms on origins of replication early in G1 (Stillman, 1996; Ritzi and Knippers, 2000; Stoeber et al., 2001), and we have used its expression as a marker for cells that are resting in G1 between cell divisions. We show that both slowly cycling NSCs and rapidly cycling proliferative progenitors express $\mathrm{Mcm} 2$, but that these populations can be distinguished by continuous labeling with nucleoside analog over a period sufficient to label only the proliferative progenitor component. Second, the nucleoside analogs 5-iodo-2'-deoxyuridine (IdU) and 5-chloro-2'-deoxyuridine (CldU), which can be independently detected, are used in double labeling experiments to establish whether cells that retain label over long periods eventually re-enter the cell cycle. These studies also confirm that $\mathrm{Mcm} 2$ is expressed in the fraction of long-term label retaining cells that re-enter the cell cycle as demonstrated by double label incorporation. Based on these methods for distinguishing NSCs from proliferative progenitors, we have characterized the expression of several putative neural stem cell markers in each cell class.

Additionally, changes in the NSC and proliferative progenitor compartments as a function of age were measured, and results from these in situ studies were compared with in vitro neurosphere-forming assays. These studies demonstrate that both NSCs and proliferative progenitors decline proportionately as a function of age in mice.

\section{Materials and Methods}

Nucleoside analog administration. All procedures involving animals were approved by the Institutional Animal Care and Use Committee. C57BL/6J male mice, of the ages indicated in the text, were used in all experiments. The double nucleoside analog-labeling scheme used CldU (Sigma, St. Louis, MO) and IdU (Sigma). CldU or IdU were administered in the drinking water at $1 \mathrm{mg} / \mathrm{ml}$ or injected subcutaneously at $10 \mathrm{mg} / \mathrm{ml}$ in $0.9 \%$ saline as indicated. In the case of the long-term-labeling experiment, CldU was administered in the drinking water for 3 weeks, followed by 1 week in the absence of label, and administration of IdU in the drinking water for an additional 3 weeks. Short-term labeling experiments included $3 \mathrm{~d}$ administration of IdU in the drinking water and one injection of $200 \mu \mathrm{l} \mathrm{CldU}$ solution $2 \mathrm{hr}$ before killing. Young and old animals consumed similar amounts of drinking water (0.19 $\mathrm{ml} \cdot \mathrm{gm}^{-1} \cdot \mathrm{d}^{-1}$ for young and $0.17 \mathrm{ml} \cdot \mathrm{gm}^{-1} \cdot \mathrm{d}^{-1}$ for old animals).

Ara-C infusion. Mice were anesthetized with a mixture of ketamine (10 $\mathrm{mg} / \mathrm{ml})$ and xylazine $(1 \mathrm{mg} / \mathrm{ml})$ administered intraperitoneally at 10 $\mu \mathrm{l} / \mathrm{gm}$ and placed in a Kopf stereotaxic frame (David Kopf Instruments, Tujunga, CA). A midline incision was made in the sterilely prepared scalp, and a $1 \mathrm{~mm}$ burr hole was drilled at $-0.8 \mathrm{~mm}$ lateral on the bregma suture. A $2 \%$ Ara-C (Sigma) solution in $0.9 \%$ saline was delivered into the lateral ventricle through an implanted cannula (Brain Infusion Kit; Durect, Cupertino, CA). Micro-osmotic pumps (model 1007D; flow rate $0.5 \mu \mathrm{l} / \mathrm{hr}, 7 \mathrm{~d}$; Durect) attached to the cannula and loaded with drug solution were implanted subcutaneously. Animals were killed immediately after $7 \mathrm{~d}$ of continuous Ara-C infusion. Mice received IdU in their drinking water for $3 \mathrm{~d}$ and one injection of $200 \mu$ l CldU solution $2 \mathrm{hr}$ before killing.

Tissue preparation and immunostaining. Animals were killed by cerebrospinal dislocation and perfused with $4 \%$ paraformaldehyde in PBSA. Brains were isolated and postfixed in the same solution overnight. After paraffin embedding, $7-\mu \mathrm{m}$-thick sections were dewaxed in xylene, rehydrated in a series of ethanol solutions, incubated in $0.01 \mathrm{M}$ citrate buffer, $\mathrm{pH}$ 6.0, for $10 \mathrm{~min}$ at $100^{\circ} \mathrm{C}$, washed twice in distilled water, immersed in PBSA, pH 7.2, for $10 \mathrm{~min}$, and incubated with goat polyclonal primary anti-Mcm2 antibodies (1:250; Santa Cruz Biotechnology, Santa Cruz, CA), rabbit polyclonal anti-Musashi (1:200; Chemicon, Temecula, CA), mouse monoclonal anti-Nestin (1:200; Chemicon), and mouse monoclonal anti-GFAP (1:200; Oncogene Sciences, Uniondale, NY) in the combinations indicated in the text. Primary antibodies were applied overnight at $4^{\circ} \mathrm{C}$ followed by FITC-conjugated (1:500; Jackson ImmunoResearch, West Grove, PA) or Alexa Fluor 488-conjugated (1:500; Molecular Probes, Eugene, OR) anti-goat, Alexa Fluor 488 or 594 antimouse (1:500; Molecular Probes), or Alexa Fluor 594 anti-rabbit (1:500; Molecular Probes) secondary antibodies as appropriate for $2 \mathrm{hr}$ at room temperature. Microscopic images were acquired using a $20 \times$ objective and CCD camera attached to a Nikon Optiphot microscope. Slides were then immersed in $1.5 \mathrm{M} \mathrm{HCl}$ for $1 \mathrm{hr}$ at room temperature, washed in PBSA, treated with $0.25 \%$ trypsin for $15 \mathrm{~min}$, and stained for CldU and IdU as previously described (Aten et al., 1994). Briefly, slides were incubated overnight with mouse monoclonal anti-BrdU antibodies (1:50; cross-reactivity with IdU; Becton Dickinson, Mountain View, CA) at $4^{\circ} \mathrm{C}$, washed in Tris buffer, $\mathrm{pH} 8.0$, containing $0.5 \mathrm{M} \mathrm{NaCl}$ and $1 \%$ Tween 20 for $20 \mathrm{~min}$ at room temperature to eliminate background and possible cross-reactivity with CldU, and incubated overnight with rat monoclonal anti-BrdU antibodies (1:250; cross-reactivity with CldU; Accurate Chemicals, Westbury, NY) at $4^{\circ} \mathrm{C}$. Secondary anti-mouse Texas Redconjugated and anti-rat FITC-conjugated antibodies (1:500; both from Jackson ImmunoResearch) were applied for $2 \mathrm{hr}$ at room temperature. After counterstaining with DAPI, digital images were taken in the same locations as for Mcm2. Sets of digital images were combined and processed with Adobe Photoshop 5.5 software.

Quantification of labeled cells and statistical analysis. The lateral wall of the lateral ventricle was analyzed for both ventricles of three mice per age group in each experiment. A minimum of 10 frontal sections, taken between the genu of corpus callosum and anterior commissure, were assessed for each animal. Cells were scored on the basis of staining for $\mathrm{Mcm} 2$, CldU, and/or IdU in their nuclei. Stained cells within the SVZ were counted and expressed as the number of cells per $1 \mathrm{~mm}$ along the ventricle for each image. SEM reflects variance between ventricular sections.

Neurosphere culture. The brains obtained from 2- and 21-month-old mice were dissociated in $0.25 \%$ trypsin solution in DMEM. One-forth of the recovered cells from each brain were seeded into two 96 well microtitro plates (nontissue culture grade; ICN Biomedicals, Aurora, $\mathrm{OH}$ ) containing culture media. The culture media was DMEM-F-12 (Invitrogen, Gaithersburg, MD) supplemented with FGF-2 (20 ng/ml; Sigma), EGF (20 ng/ml; Peprotech, Rocky Hill, NJ), B27 supplement (Invitrogen), glutamine (2 mM; Invitrogen), and gentamycin ( $50 \mu \mathrm{g} / \mathrm{ml}$; Invitrogen). Media was changed on days 2 and 4 after seeding. On day 9 wells were scored for the presence of neurospheres.

\section{Results}

Mcm2 staining allows detection of a population of SVZ cells that cycle more slowly than proliferative progenitors

To determine if $\mathrm{Mcm} 2$ expression can be detected in cells that are cycling slowly within the SVZ (putative NSCs), mice were administered IdU for a period of $3 \mathrm{~d}$, and the expression of $\mathrm{Mcm} 2$ and IdU incorporation were compared. Nearly all cells that have incorporated IdU also expressed $\mathrm{Mcm} 2$ (Fig. 1a). Additionally, $\mathrm{Mcm} 2$-expressing cells that have not divided over this period were found distributed along the SVZ, as evidenced by the lack of incorporation of the nucleoside analog IdU (Fig. 1a, insets). Occasional cells that labeled with IdU but do not express $\mathrm{Mcm} 2$ were also observed and are likely to represent cells that have withdrawn from the cell cycle (see below).

To validate that expression of $\mathrm{Mcm} 2$ in the noncycling cells allows detection of NSCs, advantage was taken of the previous observation that administration of Ara-C for 1 week is sufficient to kill the majority of proliferative progenitors (Doetsch et al., 1999b). Because of their slower cycling time, the majority of NSCs are predicted to survive this treatment. To determine if the stem cells that survive an Ara-C treatment express $\mathrm{Mcm} 2$, a solution of Ara-C was applied locally into the lateral ventricles of 2-month-old mice through an implanted cannula connected to a subcutaneous micro-osmotic pump. Three days before the end of 
Ara-C treatment, mice were given IdU through their drinking water and a single injection of CldU $2 \mathrm{hr}$ before the end of the experiment. Animals were killed after 1 week of continuous Ara-C administration and compared with controls, which received IdU and CldU only.

$\mathrm{IdU}$ and $\mathrm{Mcm} 2$ staining were assessed for both control (Fig. 1a) and Ara-Ctreated (Fig. $1 b$ ) animals. As expected from previous studies, Ara-C caused a dramatic decline in the number of cells incorporating IdU over $3 \mathrm{~d}$. (A similar decline in CldU labeled cells was observed, and all IdU-labeled cells were also labeled with CldU, demonstrating that IdU labeling through the drinking water was effective in Ara-C-treated animals.) Nonetheless, $\mathrm{Mcm} 2+$ cells that were not labeled by either IdU or CldU were found distributed along the SVZ in the Ara-C-treated mice. These cells were located in area immediately adjacent to, but not within, the ependymal layer. The distribution and location of $\mathrm{Mcm} 2+$ cells in the Ara-Ctreated animals is similar to that of the $\mathrm{Mcm} 2+$ cells that fail to incorporate IdU over $3 \mathrm{~d}$ in control animals (highlighted in the insets of Fig. 1a). These observations demonstrate that $\mathrm{Mcm} 2$ detects a subset of quiescent cells that are potentially competent to proliferate and, in conjunction with 3 d continuous IdU labeling, allow NSC $(\mathrm{Mcm} 2+/ \mathrm{IdU}-)$ and proliferative progenitor $(\mathrm{Mcm} 2+/ \mathrm{IdU}+)$ SVZ compartments to be distinguished.

To characterize NSC and proliferative progenitor compartments further, the expression of Nestin (Fig. 1c,d), Musashi (Fig. 1e,f), and GFAP (Fig. 1g,h) were examined in conjunction with $\mathrm{Mcm} 2$ and IdU in both nontreated and Ara-C treated (Fig. $1 d, f, h$, insets) mice. As observed previously (Doetsch et al., 1997; Kaneko et al., 2000), all three markers are expressed in the region of the SVZ. Strong Nestin expression was present in the ependymal layer and a subset of IdU labeled SVZ cells. Nestin was not, however, observed in $\mathrm{Mcm} 2+/ \mathrm{IdU}-$ cells (putative NSCs) (Fig. $1 c, d$, arrows). Musashi is not expressed in the ependymal layer but is detected in most IdU + SVZ cells. Mcm2+/IdU-SVZ cells also express Musashi. These observations suggest that Musashi is expressed in the majority of both NSCs and proliferative progenitors. However, Musashi expression was also observed in Mcm2-/IdU - cells within the corpus callosum and other locations (data not shown). To determine if Nestin and Musashi detect different subsets of proliferative progenitors, double staining for these markers was performed as shown in
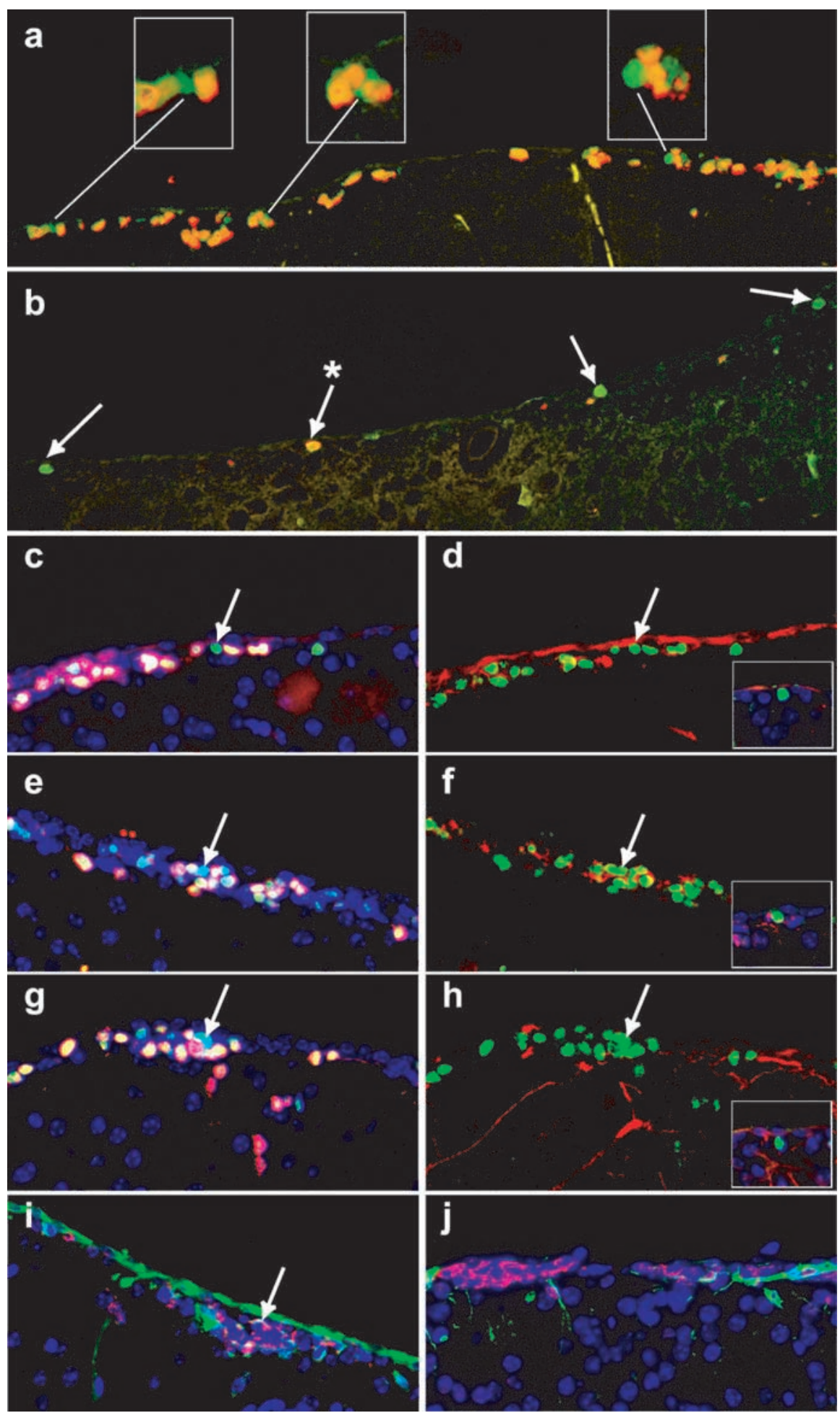

Figure 1. Cells expressing $\mathrm{Mcm} 2$ and that failed to divide over $3 \mathrm{~d}$ are present within the SVZs of untreated and Ara-C-treated mice. a shows SVZs from a 4.5-month-old mouse administered IdU for $3 \mathrm{~d}$ in their drinking water and stained (20-22) for Mcm2 (green) and IdU (red). The majority of cells stain for both $\mathrm{Mcm} 2$ and IdU and appear orange. Insets in $a$ show nuclei staining for $\mathrm{Mcm} 2$ only. $b$ shows an SVZ from a mouse treated with Ara-C for $7 \mathrm{~d}$, administered IdU for the final $3 \mathrm{~d}$ of Ara-C treatment, and stained for $\mathrm{Mcm} 2$ and IdU where the effect of Ara-C administration was assessed on the contralateral side from the site of cannula implantation. Arrows indicate Mcm2-positive/ldU-negative nuclei, and the asterisk indicates an Mcm2-positive/IdU-positive nucleus. $c-h$ show the expression of Nestin $(c, d)$, Musashi $(e, f)$, and GFAP $(g, h)$ in Mcm2/IdU-stained sections from 4.5-monthold mice, whereas the insets in $d, f$, and $h$ show expression of these markers in Mcm2-positive cells in Ara-C-treated mice. Signals from Mcm2 (green), IdU (red), and DAPI (blue) are shown in c, $e$, and $g$, and signals from Mcm2 (green) and Nestin (red), Musashi (red) or GFAP (red) are shown in $d, f$, and $h$, respectively. Arrows in $c-h$ indicate Mcm2 $+/ / \mathrm{IdU}-$ nuclei. $i$ and $j$ show double staining with Nestin (green) and Musashi (red) or GFAP (green) and Musashi (red), respectively, whereas the arrow in $i$ indicates colocalization. 

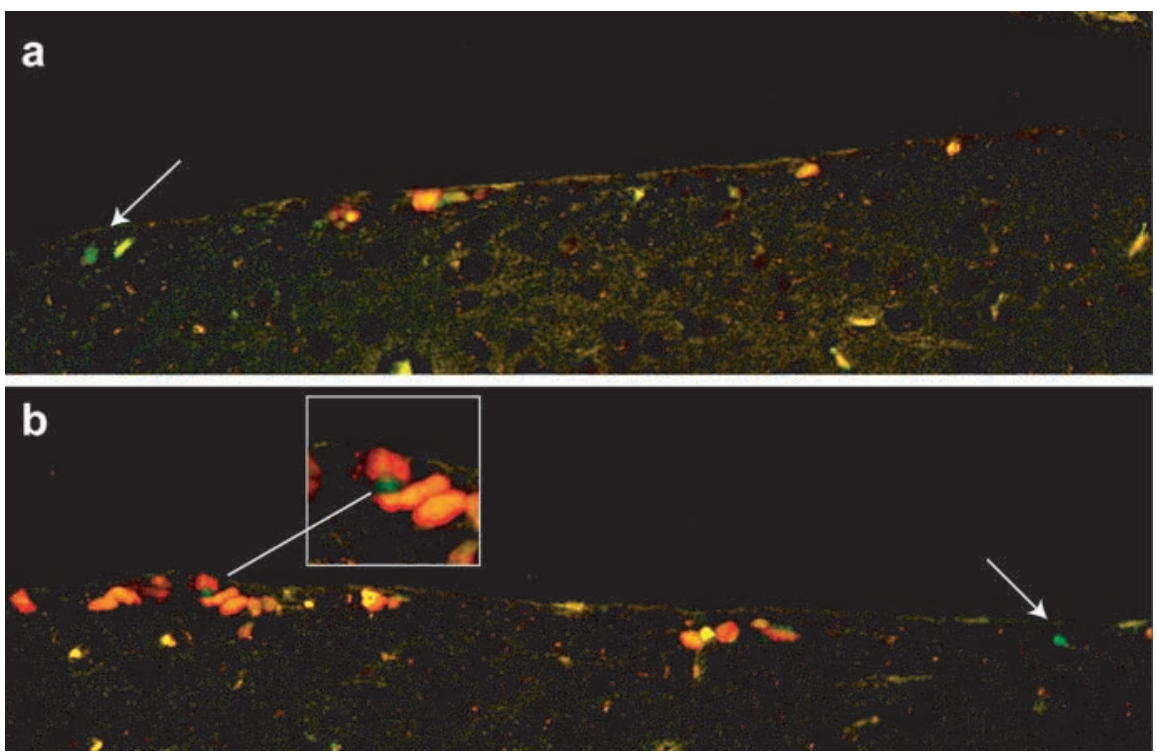

Figure 2. Rapidly dividing cells and cells that failed to divide within $3 \mathrm{~d}$ but express Mcm2 in the SVZs of old mice. For comparison with young mice (Fig. 1a), 26-month old mice were administered IdU for $3 \mathrm{~d}$ and stained for both Mcm2 (green) and IdU (red). $a$ and $b$ are from two different regions of the same mouse and show the range of staining patterns observed. Arrows indicate $\mathrm{Mcm} 2$-positive, IdU-negative cells that are not associated with IdU-stained cells. The inset in b shows an Mcm2-positive, IdU-negative cell that is associated with IdU-stained cells similar to those observed in younger mice.

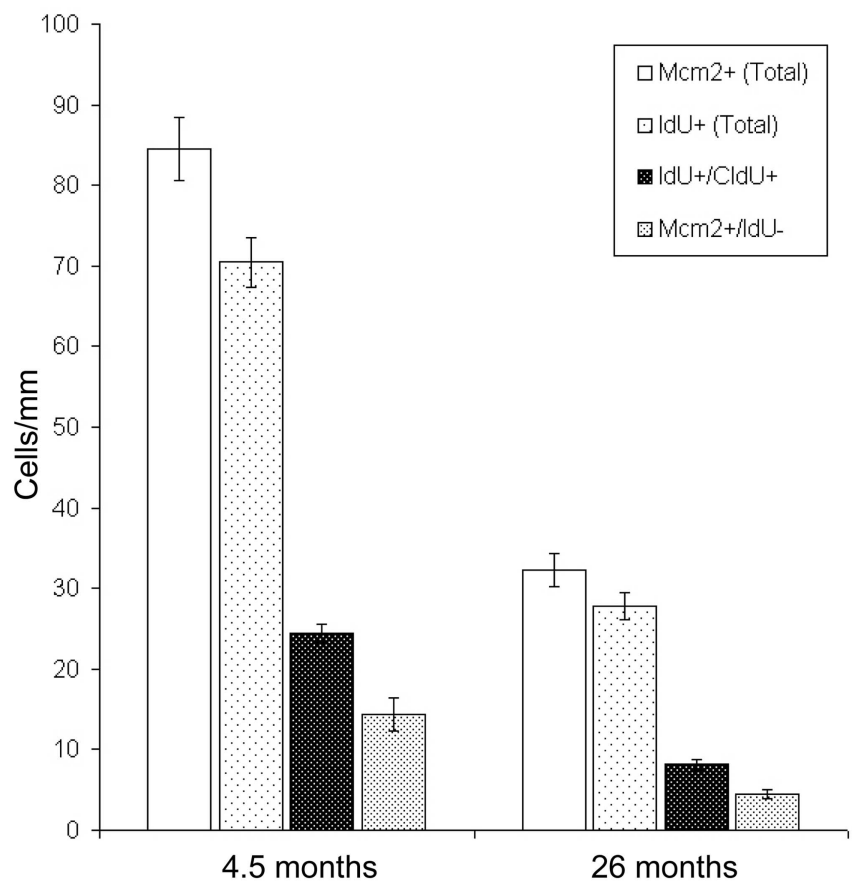

Figure 3. Different classes of cells detected within the SVZ decline in parallel in older mice. 4.5-month-old and 26-month-old mice were administered IdU for $3 \mathrm{~d}$ and injected with a pulse of $200 \mu \mathrm{l}$ CldU solution in $0.9 \%$ saline $(10 \mathrm{mg} / \mathrm{ml}) 2 \mathrm{hr}$ before the end of the experiment (to assess the fraction of cells in S-phase). The number of total $\mathrm{Mcm} 2$-positive cells, total IdUpositive cells, the fraction of IdU-positive cells that also stain for CldU, and the fraction of $\mathrm{Mcm} 2$-positive cells that do not stain for IdU was determined.

Figure 1i. Cells expressing each of the markers alone and cells in which the markers colocalize were found (Fig. $1 i$, arrow).

GFAP expression (Fig. $1 g, h$ ) was more difficult to evaluate relative to $\mathrm{Mcm} 2$ because GFAP-positive processes extended throughout much of the SVZ. However, double staining for GFAP and
Musashi, which is associated with both NSC and proliferative progenitor compartments, demonstrates no overlap between GFAP and Musashi expression.

\section{Neural stem cells and proliferative progenitors decline proportionately with age}

Based on the above observations, $\mathrm{Mcm} 2$ and IdU staining after $3 \mathrm{~d}$ of IdU administration were used to compare NSCs and proliferative progenitors in the SVZs of 4and 26-month-old mice. Additionally, mice were given a pulse of CldU, by injection, $2 \mathrm{hr}$ before the end of the experiment to allow estimation of the proportion cells that were in S-phase at the end of the experiment. The appearance of IdU plus Mcm2-stained SVZs from the 4-monthold animals is shown in Figure $1 a$. The SVZs from the 26-month-old animals are shown in Figure 2, $a$ and $b$, and in contrast to the younger mice exhibit an overall reduction in the level of IdU incorporation. In many regions few IdU-labeled cells were present (Fig. 2a), whereas in others clusters of staining cells were present (Fig. 2b) that resembled those found in younger animals. Quantification of the number of IdU-labeled cells per millimeter of SVZ is shown in Figure 3. If the number in young animals is taken as $100 \%$, this number had declined to $39 \%$ at 26 months. Because IdU incorporation over a $3 \mathrm{~d}$ period should label virtually all of the proliferative progenitors, the total number of proliferative progenitors in the older mice has declined to less than half that in the young animals.

Similar to young animals, Mcm2-positive cells that had failed to replicate within the $3 \mathrm{~d}$ IdU-labeling period were observed in old mice. These were present in many of the clusters of IdUlabeled cells, as seen in young mice (Fig. $2 b$, inset). Mcm2positive, IdU-negative cells that were not associated with IdUlabeled cells were also observed at intervals in the 2-year-old animals (Fig. 2a, $b$, arrows). However, the total number of $\mathrm{Mcm} 2-$ positive/IdU-negative cells in 2-year-old animals is less, and the cells are sparser than in the younger animals. Quantification of these cells demonstrates that they decline approximately in parallel to the number of proliferative progenitors (Fig. 3). In contrast, the fraction of the proliferative progenitors that are in $S$-phase at any given time, as measured by the proportion of IdU-labeled cells that had incorporated CldU, was not significantly changed between the youngest and oldest animals (36.0 \pm $1.4 \%$ vs $33.6 \pm 2.3 \%$ ), demonstrating that these cells do not change their rate of division during aging.

The parallel decrease in $\mathrm{Mcm} 2$-positive cells that do not divide within $3 \mathrm{~d}$ suggests that the stem cell population is declining in proportion to the overall decline in neurogenesis in older animals and is sufficient to account for this decline with age. The major caveat to this conclusion is the possibility that quiescent NSCs can rest in a state in which $\mathrm{Mcm} 2$ is not expressed and $\mathrm{Mcm} 2$ expression is not allowing detection of all neural stem cells.

\section{Nucleoside analog double labeling confirms that stem cells decline with age}

As an alternative method to establish whether the number of NSCs declines with age, a double nucleoside-labeling scheme was 
developed to establish the fraction of longterm-label retaining cells that remaining in the cell cycle. Additionally, to confirm the utility of $\mathrm{Mcm} 2$ as a marker for slowly cycling cells, the expression of $\mathrm{Mcm} 2$ in long-term-label retaining cells remaining in the cell cycle was compared with those that ceased division. For this experiment, mice were given the nucleoside analog CldU in their drinking water for 3 weeks as a first marker for replicating cells. After a period of 1 week in the absence of label, the animals were administered the second nucleoside analog IdU, which can be detected separately from CldU (Fig. $4 a-d$ ), for an additional 3 weeks. During the initial labeling period with CldU, both stem cells and proliferative progenitors will become labeled. During the 4 week period after CldU administration, this label will be diluted in rapidly dividing cells. Two classes of cells could retain label: slowly dividing stem cells or cells that have ceased to divide (either as consequence of differentiation in situ or because of nucleoside analog toxicity). Because IdU is present during the final 3 weeks of the experiment, its incorporation is expected in all dividing cells, including stem cells. This strategy should allow the subset of long-term label retaining cells that are stem cells to be positively identified.

The paradigm described above was used for labeling both young ( 2 month) and old (24 month) animals. Consistent with the $3 \mathrm{~d}$ labeling experiment, an obvious difference was found in the number and distribution of IdU-labeled cells between the young and old animals. Cells that had incorporated IdU were found throughout the SVZs of young animals (Fig. $4 g$ ). In contrast, the number of IdU-labeled cells in the SVZs of old animals was reduced, and the labeled cells that were present were not evenly distributed (Fig. 4h). Cells staining for CldU incorporation are also shown in Figure $4, g$ and $h$. As for IdU, the frequency with which CldU-marked cells were found in 24-month-old animals is reduced from that found in the 2-month-old animals. The proportion of CldU-labeled cells that had undergone additional divisions, as evidenced by the presence of both CldU and IdU, was also determined. Although there are fewer CldU-labeled cells in the older animals, for both sets of animals $\sim 60 \%$ of the cells that had incorporated CldU also stained for IdU (Fig. 5). The intensity of CldU staining is also higher in those cells that failed to incorporate IdU, consistent with the absence of additional divisions after the initial CldU incorporation. Furthermore, 96\% of cells incorporating only CldU were also negative for expression of the G1 phase marker $\mathrm{Mcm} 2$. These observations suggest that these cells have permanently withdrawn from the cell cycle.

$\mathrm{Mcm} 2$ expression was also examined in the CldU/IdU doublelabeled cells. Two-thirds of these cells express Mcm2 (Figs. 4e,f, insets, 5), which is expected for slowly cycling stem cells that are resting in the G1-phase of the cell cycle. Although this ratio was similar between young and old animals, the number of CldU/ IdU/Mcm 2 triple-labeled cells in old mice is approximately half the number in young mice. This population of cells is likely to represent the majority of cycling NSCs and is reduced in parallel with the overall decline in proliferating cells. Classification of the remaining one-third of the CldU/IdU double-labeled cells that do not express $\mathrm{Mcm} 2$ is less certain. One possibility is that they are differentiated cells that have withdrawn from the cell cycle. However, they retain sufficient levels of CldU to be detected, hence, they cannot have undergone more than a few additional divisions beyond the initial CldU labeling cycle before differentiation. A second possibility is that CldU incorporation is toxic to a subset of cells (Brandon et al., 2000). Alternatively, a fraction of these cells may be stem cells that are at a point in the cell cycle where $\mathrm{Mcm} 2$ expression is absent or undetectable. In any case, two-thirds of the CldU/IdU-positive cells are $\mathrm{Mcm} 2$-positive in both young and old animals demonstrating that Mcm 2 expression allows detection of the majority of neural stem cells. Hence, to the extent that the fraction of CldU/IdU-labeled cells that are expressing $\mathrm{Mcm} 2$ represents the distribution of the stem cells within different phases of the cell cycle, we can conclude that this parameter does not differ between young and old animals.

A small number of $\mathrm{Mcm} 2$-stained cells that were not positive for CldU or IdU were also observed. Such cells were generally not associated with clusters of IdU-labeled cells (data not shown). Similar Mcm2 +/IdU - cells, not associated with clusters of dividing cells, were observed previously in the $3 \mathrm{~d}$ IdU-labeling experiment (Fig. 2, arrows). This observation suggests the presence of a minor population of cells that are arrested in the cell cycle.

In summary, we can conclude that the overall age-related reduction in CldU/IdU/Mcm2 triple-labeled cells in this experiment is consistent with results from the short-term labeling experiment, and together these results demonstrate that NSCs in old animals are reduced to less than half their number in young animals.

\section{Neurosphere formation is reduced in explants from old animals}

To determine if the reduction in proliferating cells detected in situ would also be reflected in the efficiency with which neurospheres are recovered in vitro, cells from the brains from young (2 month) 


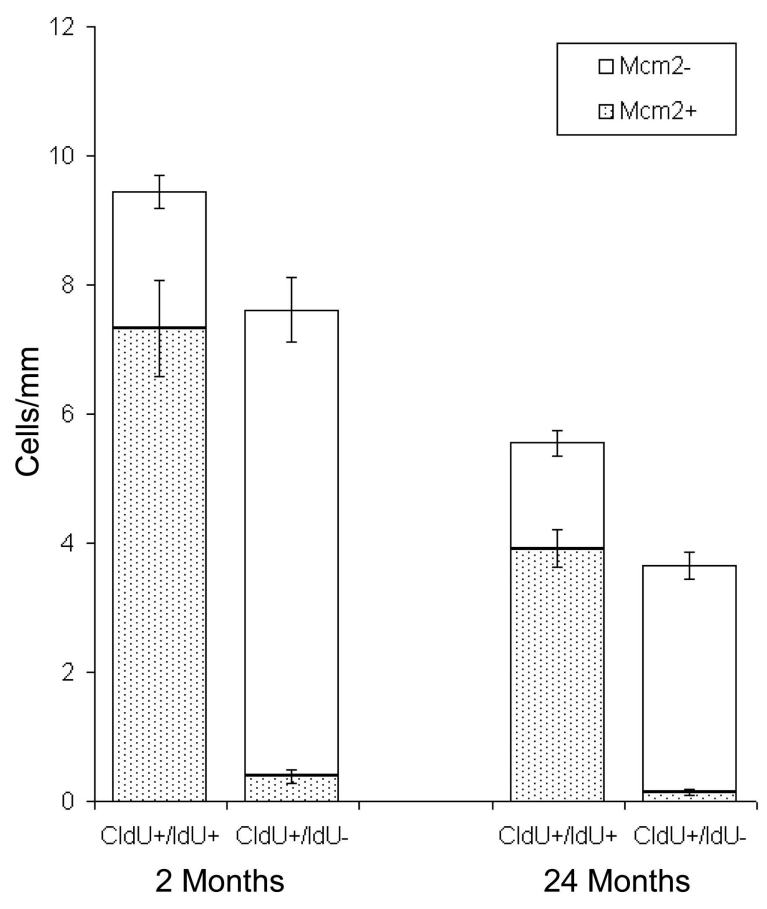

Figure 5. Different classes of long-term label retaining decline proportionately with age. Cells staining for CldU, IdU, and $\mathrm{Mcm} 2$ or combinations, from the experiment shown in Figure 4, were quantified as a function of distance in millimeters of SVZ for young and old mice. CIdUpositive cells (i.e., cells that divided during the first 3 week labeling period and retained label for at least 4 additional weeks) that express IdU (i.e., divided again during the second 3 week labeling period at the end of the experiment) or do not express IdU (i.e., failed to divide during the second 3 week labeling period) are shown for both 2-month-old and 24-month-old mice. Additionally, each of these cells was scored for expression of $\mathrm{Mcm} 2$, and the stippled portion of the bar indicates the fraction of each class that expressed $\mathrm{Mcm} 2$.

and old (21 month) mice were dissected and prepared for culture in parallel, as described in Materials and Methods. Cells from each brain were distributed into 192 individual microtiter wells and cultured for $9 \mathrm{~d}$ before counting the number of wells containing neurospheres. Three separate experiments were performed and averaged. In each case the number of wells containing neurospheres in cultures derived from the brains of old mice was reduced to approximately half the level $(51.1 \pm 3.9 \%)$ found for young mice.

To insure that the initial seeding density of cells derived from young and old animals did not contribute to this difference, a fourth experiment was performed in which the number of viable cells was determined (young, $\sim 4.3 \times 10^{7}$ /brain; old $\sim 4.9 \times$ $10^{7} /$ brain) before culture in microtiter plates. In this experiment both the number of wells containing neurospheres (young, 108; old, 64) and the total number of neurospheres (young, 150; old, 83 ) were counted and demonstrate that the reduction in old animals does not result from differences in the initial seeding density. These observations are consistent with the age-dependent reduction in NSC and proliferative progenitor number in situ documented above.

\section{Discussion}

One method used to detect NSCs in the present study relies on the expression of $\mathrm{Mcm} 2$ in these cells. $\mathrm{Mcms}$, origin recognition complex (ORC), and Cdc6 proteins form pre-replicative complexes on origins of replication in the G1 phase of the cell cycle, and their presence maintains the origin in a state in chromatin that is competent for initiation of replication (Stillman, 1996;
Ritzi and Knippers, 2000; Stoeber et al., 2001). These properties suggested that $\mathrm{Mcm} 2$ could be a useful marker for slowly cycling cells, which generally rest in G1 between cell divisions. Consistent with this possibility, immunohistological detection of various $\mathrm{Mcm}$ proteins identifies a significantly higher fraction of cycling cells in tumors (Freeman et al., 1999) and some normal tissues (Endl et al., 2001; Stoeber et al., 2001) than are detected using conventional proliferation markers such as PCNA and Ki67. The present study confirms that, at least $\mathrm{Mcm} 2$, is present during the extended, up to $15 \mathrm{~d}$, cell cycle within the NSCs of the SVZ in mice. Because $\mathrm{Mcm} 2$ is required in all replicating cells, its expression should allow detection of somatic stem cells in a variety of tissues and is not limited to the detection of NSCs as demonstrated here.

Based on the ability to detect NSCs and distinguish them from proliferative progenitors, we have re-examined the expression of several markers that previous studies demonstrated are expressed in the SVZ to determine whether they are restricted to a specific cellular compartment. Three putative stem cell markers were examined here. First, expression of the neural-specific intermediate filament Nestin, which is expressed throughout the neuroectoderm during embryogenesis, has been demonstrated in the ependymal layer, and a subset of SVZ cells in the adult (Doetsch et al., 1997). A second marker, GFAP, is not expressed within the ependymal layer but has been localized to a partially overlapping set of SVZ cells to those expressing Nestin (Doetsch et al., 1997). Musashil is an RNA binding protein that has been shown to localize to a broad range of proliferating cells within the CNS of a variety of vertebrates (Kaneko et al., 2000). Comparison of cells expressing each of these markers with $\mathrm{Mcm} 2$-positive cells that either were, or were not, also labeled with IdU in the short-term labeling paradigm described here (as well as Mcm2-positive cells after Ara-C treatment) demonstrates that only Musashi is unequivocally expressed in the $\mathrm{Mcm} 2+/ \mathrm{IdU}-$ (NSC) fraction, and most $\mathrm{Mcm} 2+/ \mathrm{IdU}-$ staining nuclei where associated with Musashi expression in the adjacent cytoplasm. Musashi is also expressed in most $\mathrm{Mcm} 2+/ \mathrm{IdU}+$ cells. No colocalization of Nestin with $\mathrm{Mcm} 2+/ \mathrm{IdU}$ - cells was observed, although double label experiments with Nestin and Musashi demonstrate the presence of some cells that express both markers. The pattern of GFAP expression relative to $\mathrm{Mcm} 2+/ \mathrm{IdU}$ - nuclei was more difficult to establish because of the morphology of GFAP-expressing cells. Double label experiments detecting GFAP and Musashi show no overlap in the expression of these markers, which could suggest that GFAP is not expressed in the NSC compartment; however, it is also possible that GFAP and Musashi are localized to different cytoplasmic compartments.

The present study demonstrates that Nestin and, with the caveats discussed above, GFAP are not expressed in the slowly cycling NSC in situ. However, using Nestin or GFAP promoterenhancer elements to drive expression of EGFP, it has been demonstrated that cells expressing these markers are capable of giving rise to neurospheres in culture (Doetsch et al., 1999a; Kawaguchi et al., 2001). Additionally in the case of GFAP, a negative selection scheme using expression of HSVtk from the GFAP promoter demonstrated that treatment of mice carrying this construct with gancyclovir for a period of $3 \mathrm{~d}$ was sufficient to eliminate $>95 \%$ of neurosphere-forming cells when cells from the brains of such animals were subsequently placed in culture (Morshead et al., 2003). If neurospheres are derived solely from slowly cycling NSCs, these findings would conflict with the apparent lack of Nestin and GFAP expression in Mcm2 +/IdU - cells identified here. However, several observations suggest that the more 
rapidly cycling proliferative progenitor, detected as $\mathrm{Mcm} 2+$ / $\mathrm{IdU}+$ cells here, are the source of neurospheres in vitro. First, the period of gancyclovir treatment of mice carrying HSVtk driven from the GFAP promoter was only $3 \mathrm{~d}$, which is the same period of labeling used to mark rapidly dividing cells with IdU $(\mathrm{McM} 2+/ \mathrm{IdU}+$ cells $)$ in the present study and is expected to affect predominately the proliferative progenitor population. Additionally, evidence suggesting that neurospheres arise from proliferative progenitors is provided by the observation that gancyclovir treatment of mice carrying the HSVtk gene driven from the Dlx2 promoter, which is expressed specifically in the proliferating progenitor, also leads to a decline in neurosphere formation in vitro (Doetsch et al., 2002). Finally, neurosphere formation from mice treated with Ara-C is also reduced but recovers in parallel to the recovery of the proliferative progenitor population following Ara-C withdrawal (Doetsch et al., 2002). Together, these observations make a strong case that neurospheres formed in vitro are derived to a substantial extent from the in vivo proliferative progenitor population. In this case finding here that Nestin, and with the caveats discussed GFAP, are not expressed in the slowly cycling NSC population is consistent with the ability of Nestin- and GFAP-positive cells to give rise to neurospheres in vitro.

A second method used to detect NSCs in this study is based on the previous observation that stem cells retain nucleoside label for a longer period of time that proliferative progenitors. This method was used first to identify epidermal stem cells (Bickenbach, 1981; Bickenbach et al., 1986) and applied to the detection of NSCs by Johansson et al. (1999). However, a significant limitation to this methodology is that recently differentiated cells, or cells that have artifactually withdrawn from the cell cycle because of nucleoside analog toxicity, will also retain label indefinitely. Here we demonstrate that the use of a double nucleoside analoglabeling scheme allows the stem cell and differentiated cell populations to be distinguished, and this approach may be generally applicable.

The methodologies described above have been applied here to define the cellular basis for the observation that neurogenesis in 2 -year-old mice declines to approximately half the level found in 2-month-old animals (Hopewell, 1971; Tropepe et al., 1997). In the present study age-related changes in NSC and proliferative progenitor populations within the SVZs of mice were examined to establish whether the decline results from reduction in the number of stem cells or from some later step in the proliferation and differentiation of these cells. Comparison of nucleoside incorporation into the SVZs of animals of two different age groups (4- and 26-month-old) showed a decline in neurogenesis in old animals by more than a factor of 2, consistent with previous studies. Furthermore, two alternative methods demonstrate that the factor of 2 decline is reflected in both the NSC and proliferative progenitor compartments in situ. Additionally, the rate of division of the proliferative progenitors was shown to be unaffected by age. Finally, analysis of neurosphere recovery in culture using a clonogenic assay showed a factor of two decrease between young and old animals (although, if neurospheres are derived primarily from proliferative progenitors, e.g., Doetsch et al., 2002 and discussed above, this observation could reflect a decrease in both the NSC and proliferative progenitors). Together these results demonstrate that declining neurogenesis with age is a consequence of the loss of slowly cycling NSCs.

Whether the loss of stem cells contributes to the aging phenotype is an issue that has been considered for several somatic tissues previously. Within the hematopoetic system, a restriction on the replicative capacity of the stem cells has been demonstrated by serial transplantation studies (Harrison and Astle, 1982). Additionally, cell-autonomous factors have been demonstrated to affect the longevity of these cells between different strains of mice, and it has been possible to map the locations of some of the relevant genes (Geiger and Van Zant, 2002). Nonetheless, the number of hematopoetic stem cells does not appreciably decline, and in some strains may even increase, during aging in mice (Morrison et al., 1996). Hence, it has been argued that genetic changes occurring within hematopoetic stem cells primarily affect the quality of the stem cells (i.e., their ability to function) rather than the quantity (Geiger and Van Zant, 2002).

The present study demonstrates that, unlike hematopoetic stem cell, NSCs within the SVZ are lost during aging. Similar age-related declines have also been documented for epidermal stem cells (Michel et al., 1996) and skeletal muscle satellite cells (Snow, 1977; Gibson and Schultz, 1983; Dedkov et al., 2003). At least three factors could contribute to the difference between these systems and the hematopoetic system. First, the reserve capacity of the hematopoetic stem cell population may be greater. Second, the rate at which stem cells are replaced by symmetric divisions, resulting in two daughter stem cells may be higher for hematopoetic stem cells. Finally, there may be tissue-specific differences in the ability of the stem cells to respond to genetic damage, which affects whether the cells survive and accumulate damage or undergo apoptosis. Regardless of whether the outcome is the functional or actual depletion of stem cells, genetic damage within each of these stem cell compartments is likely to be a primary cause of aging-related phenotypes (Donehower, 2002).

To the extent that neurogenesis within the adult contributes to the maintenance of normal CNS function, the loss of functioning NSCs can be expected to lead to age-related neurodegeneration (Armstrong and Barker, 2001; Shors et al., 2001). This neurodegeneration is a potential contributing factor to the development of Parkinson's and Alzheimer's diseases, each of which has strong age dependence in its onset. Stimulation of more rapid NSC division could in part compensate for the reduced number of functioning cells and is a potential therapeutic approach. However, a potential side effect of this approach that will need to be addressed is the possibility that such therapies also lead to an increase in the rate at which NSCs are lost because of replication-related errors.

\section{References}

Altman J, Das GD (1965) Autoradiographic and histological evidence of postnatal hippocampal neurogenesis in rats. J Comp Neurol 124:319-335

Armstrong RJ, Barker RA (2001) Neurodegeneration: a failure of neuroregeneration? Lancet 358:1174-1176.

Aten JA, Stap J, Hoebe R, Bakker PJ (1994) Application and detection of IdUrd and CldUrd as two independent cell-cycle markers. Methods Cell Biol 41:317-326.

Bickenbach JR (1981) Identification and behavior of label-retaining cells in oral mucosa and skin. J Dent Res 60:1611-1620.

Bickenbach JR, McCutecheon J, Mackenzie IC (1986) Rate of loss of tritiated thymidine label in basal cells in mouse epithelial tissues. Cell Tissue Kinet 19:325-333.

Brandon ML, Mi L, Chaung W, Teebor G, Boorstein RJ (2000) 5-chloro-2' deoxyuridine cytotoxicity results from base excision repair of uracil subsequent to thymidylate synthase inhibition. Mutat Res 459:161-169.

Dedkov EI, Borisov AB, Wernig A, Carlson BM (2003) Aging of skeletal muscle does not affect the response of satellite cells to denervation. J Histochem Cytochem 51:853-863.

Doetsch F, Garcia-Verdugo JM, Alvarez-Buylla A (1997) Cellular composition and three-dimensional organization of the subventricular germinal zone in the adult mammalian brain. J Neurosci 17:5046-5061. 
Doetsch F, Caille I, Lim DA, Garcia-Verdugo JM, Alvarez-Buylla A (1999a) Subventricular zone astrocytes are neural stem cells in the adult mammalian brain. Cell 97:703-716.

Doetsch F, Garcia-Verdugo JM, Alvarez-Buylla A (1999b) Regeneration of a germinal layer in the adult mammalian brain. Proc Natl Acad Sci USA 96:11619-11624.

Doetsch F, Petreanu L, Caille I, Garcia-Verdugo JM, Alvarez-Buylla A (2002) EGF converts transit-amplifying neurogenic precursors in the adult brain into multipotent stem cells. Neuron 36:1021-1034.

Donehower LA (2002) Does p53 affect organismal aging? J Cell Physiol 192:23-33.

Endl E, Kausch I, Baack M, Knippers R, Gerdes J, Scholzen T (2001) The expression of Ki-67, MCM3, and p27 defines distinct subsets of proliferating, resting, and differentiated cells. J Pathol 195:457-462.

Eriksson PS, Perfilieva E, Bjork-Eriksson T, Alborn AM, Nordborg C, Peterson DA, Gage FH (1998) Neurogenesis in the adult human hippocampus. Nat Med 4:1313-1317.

Freeman A, Morris LS, Mills AD, Stoeber K, Laskey RA, Williams GH, Coleman N (1999) Minichromosome maintenance proteins as biological markers of dysplasia and malignancy. Clin Cancer Res 5:2121-2132.

Geiger H, Van Zant G (2002) The aging of lympho-hematopoietic stem cells. Nat Immunol 3:329-333.

Gibson MC, Schultz E (1983) Age-related differences in absolute numbers of skeletal muscle satellite cells. Muscle Nerve 6:574-580.

Harrison DE, Astle CM (1982) Loss of stem cell repopulating ability upon transplantation. Effects of donor age, cell number, and transplantation procedure. J Exp Med 156:1767-1779.

Hopewell JW (1971) A quantitative study of the mitotic activity in the subependymal plate of adult rats. Cell Tissue Kinet 4:233-238.

Johansson CB, Momma S, Clarke DL, Risling M, Lendahl U, Frisen J (1999) Identification of a neural stem cell in the adult mammalian central nervous system. Cell 96:25-34.

Kaneko Y, Sakakibara S, Imai T, Suzuki A, Nakamura Y, Sawamoto K, Ogawa Y, Toyama Y, Miyata T, Okano H (2000) Musashil: an evolutionally conserved marker for CNS progenitor cells including neural stem cells. Dev Neurosci 22:139-153.

Kawaguchi A, Miyata T, Sawamoto K, Takashita N, Murayama A, Akamatsu W, Ogawa M, Okabe M, Tano Y, Goldman SA, Okano H (2001) NestinEGFP transgenic mice: visualization of the self-renewal and multipotency of CNS stem cells. Mol Cell Neurosci 17:259-273.

Kukekov VG, Laywell ED, Suslov O, Davies K, Scheffler B, Thomas LB, O’Brien TF, Kusakabe M, Steindler DA (1999) Multipotent stem/progenitor cells with similar properties arise from two neurogenic regions of adult human brain. Exp Neurol 156:333-344.
Michel M, Torok N, Godbout MJ, Lussier M, Gaudreau P, Royal A, Germain L (1996) Keratin 19 as a biochemical marker of skin stem cells in vivo and in vitro: keratin 19 expressing cells are differentially localized in function of anatomic sites, and their number varies with donor age and culture stage. J Cell Sci 109:1017-1028.

Morrison SJ, Wandycz AM, Akashi K, Globerson A, Weissman IL (1996) The aging of hematopoietic stem cells. Nat Med 2:1011-1016.

Morshead CM, Reynolds BA, Craig CG, McBurney MW, Staines WA, Morassutti D, Weiss S, van der Kooy D (1994) Neural stem cells in the adult mammalian forebrain: a relatively quiescent subpopulation of subependymal cells. Neuron 13:1071-1082.

Morshead CM, Craig CG, van der Kooy D (1998) In vivo clonal analyses reveal the properties of endogenous neural stem cell proliferation in the adult mammalian forebrain. Development 125:2251-2261.

Morshead CM, Garcia AD, Sofroniew MV, van Der Kooy D (2003) The ablation of glial fibrillary acidic protein-positive cells from the adult central nervous system results in the loss of forebrain neural stem cells but not retinal stem cells. Eur J Neurosci 18:76-84.

Pencea V, Bingaman KD, Freedman LJ, Luskin MB (2001) Neurogenesis in the subventricular zone and rostral migratory stream of the neonatal and adult primate forebrain. Exp Neurol 172:1-16.

Reynolds BA, Weiss S (1992) Generation of neurons and astrocytes from isolated cells of the adult mammalian central nervous system. Science 255:1707-1710

Rietze RL, Valcanis H, Brooker GF, Thomas T, Voss AK, Bartlett PF (2001) Purification of a pluripotent neural stem cell from the adult mouse brain. Nature 412:736-739.

Ritzi M, Knippers R (2000) Initiation of genome replication: assembly and disassembly of replication-competent chromatin. Gene 245:13-20.

Shors TJ, Miesegaes G, Beylin A, Zhao M, Rydel T, Gould E (2001) Neurogenesis in the adult is involved in the formation of trace memories. Nature 410:372-376

Snow MH (1977) The effects of aging on satellite cells in skeletal muscles of mice and rats. Cell Tissue Res 185:399-408.

Stillman B (1996) Cell cycle control of DNA replication. Science 274:1659-1664.

Stoeber K, Tlsty TD, Happerfield L, Thomas GA, Romanov S, Bobrow L, Williams ED, Williams GH (2001) DNA replication licensing and human cell proliferation. J Cell Sci 114:2027-2041.

Tropepe V, Craig CG, Morshead CM, van der Kooy D (1997) Transforming growth factor-alpha null and senescent mice show decreased neural progenitor cell proliferation in the forebrain subependyma. J Neurosci 17: $7850-7859$. 